\title{
Model for self-replicating, self-assembling electric circuits with self-controlled growth
}

\author{
Rojoba Yasmin $\odot$ \\ Department of Electrical and Computer Engineering, The University of Memphis, Memphis, Tennessee 38152, USA \\ Max Garzon (1) \\ Department of Computer Science, The University of Memphis, Memphis, Tennessee 38152, USA \\ Russell Deaton $\odot^{*}$ \\ Department of Electrical and Computer Engineering, The University of Memphis, Memphis, Tennessee 38152, USA
}

(Received 6 April 2020; accepted 19 June 2020; published 29 July 2020)

\begin{abstract}
Tile self-assembly models have proved to be important theoretical tools for studying nanoscale manufacturing techniques, and have provided insight into the computational capabilities of systems inspired by molecular biology. A tile assembly model (rcTAM), whose tiles are composed of simple electric circuit components, exhibits three important properties akin to those found in living organisms. First, it grows from a seed tile by self-assembly of component tiles. Second, it autonomously stops growth when a maximum size is reached, as determined by parameters associated with the tiles. Third, when the circuit assembly has reached the limit of its growth, it generates an identical copy of the original seed, which then will grow to replicate a copy itself. The size of the assembled circuit is controlled by values of the circuit components (voltage sources, resistors), and a threshold voltage for tile attachment. Since every new tile attachment instantly changes the circuit properties, such as voltage drops and currents across resistors, as well as equivalent resistances, the model exhibits instantaneous distant communication and cooperation between components, as well as dynamic behavior. Proofs are given for a bound on growth, the self-replicating property, and possible aging phenomena that produce a stable circuit population. The model might have application to electrochemical growth processes at the nanoscale, and provides insight into self-replicating systems that are not necessarily composed of organic materials. In addition, it models certain features of bioelectric networks that contribute to pattern formation in collections of cells.
\end{abstract}

DOI: 10.1103/PhysRevResearch.2.033165

\section{INTRODUCTION}

Understanding the origin of living systems requires answers to fundamental scientific questions [1-3], such as how they employ the mechanism of self-assembled, self-controlled growth to self-replicate themselves. In self-assembly [4], components construct larger, more capable systems through localized interactions. Examples in living systems are amino acids and proteins, lipids and membranes, and cells and higher organisms. As a technology, molecular self-assembly is an ongoing topic of research [5], and has primarily been applied to the growth of nanostructures. Algorithmic assembly controls growth through programed interactions between component parts, which are represented as matching glues that, for example, have been implemented with complementary DNA oligonucleotides [6,7]. Self-control limits the growth of living

\footnotetext{
*rjdeaton@memphis.edu

Published by the American Physical Society under the terms of the Creative Commons Attribution 4.0 International license. Further distribution of this work must maintain attribution to the author(s) and the published article's title, journal citation, and DOI.
}

systems with mechanisms contained within the components themselves, as well as interactions with the environment. Biological growth from proteins to higher organisms requires a source of energy to sustain. For example, bacterial colonies are limited by their nutrient supply, and, as nutrition has improved, human beings have become larger.

Here, self-replication is defined as a process in which an individual senses a mature stage in its development that triggers the growth of a copy of itself. For example, in eukaryotes, signaling pathways initiate mitosis, which is a process in which two or more daughter cells are produced by cell division from an identical parent. Von Neumann pioneered computational, artificial self-replicating systems in the 1940s, and proposed an abstract machine that has the power of replication in cellular automata $[3,8]$. Moreover, a self-replicating molecular assembler has been a dream of nanoscale engineering [9]. In molecular biology, focus has been on self-replicating systems of RNA, DNA, or proteins, with the most prominent being the RNA world hypothesis [10].

In this paper, we propose a replicating circuit tile assembly model (rcTAM) that combines self-assembly and selfreplication in a nonbiological system, where the self-assembly of ladder circuits from resistive circuit tiles is controlled by a voltage source whose consumption during growth controls 
the extent and shape of the resulting structures. In rcTAM, a growing circuit senses when growth has reached its limit, and, in response, initiates growth of a new circuit identical to itself. Moreover, all of these capabilities are achieved in relatively simple resistive circuits with diodes and voltage sources, without an appeal to molecular biology, or even organic chemistry. On the supposition that living matter selfassembled from nonliving components [2,11] (abiogenesis), many models have focused on the transition from inorganic to organic molecules. Thus, the rcTAM models a system in which many of the basic characteristics of life at the molecular level are captured (self-assembly, self-control, selfreplication), and which could be leveraged for enhanced capabilities of man-made technologies and new insights into how self-replicating systems might arise.

Though the rcTAM is not a biological system, it might be a useful abstraction of bioelectric networks among somatic cells that interact with genetic mechanisms to guide pattern and shape formation, including morphogenesis, embryogenesis, tissue regeneration, and regulation of cellular abnormalities [12-16]. Endogenous electric fields and the consequent distribution of electric potentials arise from the distribution of action potentials across cell membranes and gap junctions between cells. These bioelectric networks are ancient mechanisms to control anatomy [14]. Control over pattern and form is established through rapid feedback mechanisms that are characteristic of electrical systems [14-16]. Likewise, the rcTAM attempts to model ancient mechanisms with primitive electrical components with a threshold mechanism, as in ion channels or gap junctions. In the preceding circuit tile assembly model (cTAM) [17], the goal was to control pattern formation with the shape of a potential distribution established by a voltage source and boundary conditions. The current state (growing or terminated) of an assembly was communicated instantaneously throughout the circuit by changes in current and voltage. Electric fields in gap junctions transport materials between cells [18], and cTAMs model this type of electrochemical growth phenomenon [17]. The advantage of the cTAM models is that they are amenable to exact analysis and characterization of their properties, and thus, from a theoretical perspective, might produce better understanding of information processing in bioelectric phenomena.

The cTAM in [17,19-21] consisted of resistive circuits and voltage sources, and only achieved self-assembled and self-controlled growth. Here, it is augmented with diodes and dependent voltage sources to achieve self-replication. The goal has been to achieve the desired functionality with circuits that are as simple as possible. In Sec. II, the rcTAM model is defined. In Sec. III the self-replicative property of the rcTAM model is demonstrated. In addition, a bound on the maximum length of the assembly is derived, and an aging mechanism is addressed. Finally, some discussion of possible applications and concluding remarks are given in Sec. IV.

\section{A SELF-REPLICATING CTAM (RCTAM)}

In the circuit tile assembly model cTAM [19-21], larger circuits are self-assembled from smaller unit circuits, called circuit tiles. Combining chemically inspired glues and resistive electrical circuits, circuit tiles attach if the glues match at the attachment points and the voltage drop across the attachment points equals or exceeds a certain threshold $\tau$. As the voltage at attachment points is dissipated by resistive voltage dividers with each new tile addition, growth eventually ceases at a maximum size, and thus is self-controlled. The rcTAM augments the capabilities of the cTAM by adding diodes and dependent voltage sources to achieve self-replication.

Definition II.1 (rcTAM circuit). An rcTAM circuit is a tuple $\Psi=(N, E, C, g, \partial N)$ on a graph $(N, E)$ where $N$ denotes the set of nodes, corresponding to electrical nodes in the circuit, $E$ denotes the set of edges, $C$ is a set of circuit components (chosen from resistors, diodes and voltage sources) assigned to edges $e_{(i, j)} \in E$ where $\{i, j\} \in N$, and $g$ maps some subset of nodes $\partial N$ to some subset of glues labeled from a finite alphabet $\Sigma$, i.e., $g: \partial N \rightarrow \Sigma$. $\partial N=N_{\text {in }} \cup N_{\text {out }}$ consists of two finite subsets of nodes, input nodes $N_{\text {in }}$ and output nodes $N_{\text {out }}$ to the circuit, and are the points at which glues bind tiles together on the boundary of the circuit.

The rcTAM tiles (rcTiles) are small rcTAM circuits, and represent the most primitive, elementary circuits from which all others are assembled. Each rcTile contains a voltage divider made of resistors, as well as voltage sources and diodes. A diode is an electrical device that functions as a switch. In an ideal diode, if the voltage across it is greater than or equal to a threshold $V_{\mathrm{thr}}$, then it is forward biased and the diode acts as a perfect conductor, or short circuit with zero resistance to current flow. Otherwise, it is reverse biased and behaves as a perfect insulator, or open circuit with infinite resistance to current flow.

Definition II.2 (Replicating circuit tile assembly system). A replicating circuit tile assembly system (rcTAM) is a tuple $\mathcal{C}=(\Gamma, S, \tau, v, \zeta)$, where $\Gamma$ is a finite set of rcTiles, $S \subset \Gamma$ is a set of seed rcTiles, $\tau \in \mathbb{R}_{+}$is the threshold voltage that sets one of the criteria for further attachment, and $v \in \mathbb{R}_{+}$is the electric node potential in the circuit. $\zeta$ is a glue indicator function that indicates whether glues on input nodes of rcTiles match or bind to glues on output nodes of rcTAM circuits, or $\zeta: \Gamma\left(N_{\text {in }}\right) \times \Gamma\left(N_{\text {out }}\right) \rightarrow\{0,1\}$.

In the rcTAM, an assembly is a sequence of resistive electrical ladders that are connected, but electrically isolated from each other. Each ladder is obtained from the previous or an initial seed tile by attachment of rcTiles. A ladder that has finished growth through addition of rcTiles has reached "adulthood," and has a certain number $t$ of tiles. An index $k \in$ $\{1, \ldots, n t, \ldots\}$, indicates a specific tile in the ladder assembly, as well as a time step, where $n$ is the number of adult ladders in the current assembly. The current tile in the ladder that offers a glue for attachment (current last tile added to an assembly) is denoted $k_{\tau}$, and the voltage drop between its output nodes is the "tip" voltage of the circuit. Once started, the assembly does not stop as long as conditions for attachment are met. Because any replication process that terminates after a finite number of steps would lead to eventual extinction of that species, a prerequisite to a viable self-replication process would be one that is theoretically capable of running forever, as the rcTAM.

Voltages (potential differences between nodes) and currents in the ladder are a function of the location $k$ and tile parameters. Thus, voltages will be denoted as $V_{(i, j)}^{\gamma}(k)$, where the first node $i$ in an edge refers to the more positive 


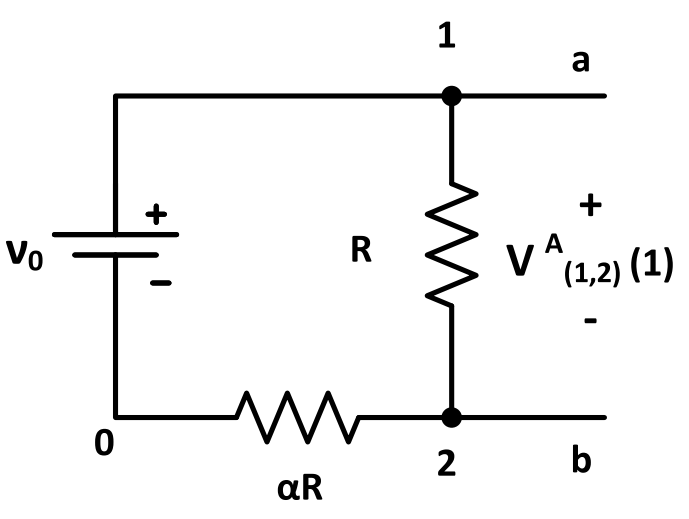

FIG. 1. Tile A (seed tile) for the rcTAM consisting of one DC voltage source $v_{0}$ and two resistors, $R$ and $\alpha R$. It has one pair of output nodes $\{1,2\}$ across the resistor $R$. Node 1 has glue $a$ and node 2 has glue $b$.

potential for a particular rcTile $\gamma \in \Gamma$. The tip voltage at the current last tile in the ladder, where a new tile attachment is determined, is denoted $V_{(i, j)}^{\gamma}\left(k_{\tau}\right)$. (The indices $\gamma$ and $k$ may be omitted when the location of the tile in the ladder is clear.) In general, two nodes $\{i, j\} \in N_{\text {out }}$ of an rcTAM circuit $\psi_{1}$ can attach to two nodes $\{l, m\} \in N_{\text {in }}$ of an rcTile circuit $\psi_{2}$ if $\zeta\left(\psi_{1}(i), \psi_{2}(l)\right)=1$ and $\zeta\left(\psi_{1}(j), \psi_{1}(m)\right)=1$, and either $V_{(i, j)}^{\gamma}\left(k_{\tau}\right)=v(i)-v(j) \geqslant \tau$ or $V_{(l, m)}^{\gamma}=v(l)-v(m) \geqslant$ $\tau$, where $V_{(l, m)}$ is a voltage across input nodes in a single rcTile (i.e., not yet part of a ladder). Ideally, growth is restricted to input nodes of rcTiles attaching to output nodes of a larger, growing rcTAM circuit. Once attached, a given input node and given output node become one node electrically, though the distinct node numbers associated with the attaching rcTiles will be retained.

If life is taken as the prototypical self-replicating system, then one usually thinks of a living entity as one that grows by consuming resources from the environment, produces signals indicating that it has reached maturity and is capable of reproduction, and then engages in processes that result in close facsimiles of itself as offspring. The rcTAM has been designed with this in mind.

Definition II.3 (Self-replication). A self-replicating rcTAM is a cTAM that grows to a maximum size, senses that this specific stage in its growth has occurred, and, in response, initiates a new process to grow another identical assembly.

To exhibit self-replication in an rcTAM, three rcTiles will be defined. For clarity, matching glues are denoted like DNA Watson-Crick complementary oligonucleotides, so that a glue $a$ matches its complement $\bar{a}$. In addition, node numbers are referenced to specific, individual rcTiles, and not the entire, growing assembly. Once the adult ladder has replicated, the tile numbering $(k)$ continues on at the seed for the new, replicated ladder. Tile $\mathrm{A}$ is the seed tile for the assembly process. There is only one per assembly at position $k=1$. It consists of one independent voltage source $\left[v_{0}=V_{(1,0)}^{A}(1)\right]$ on $e_{(1,0)}$, and two resistors with $R$ on $e_{(1,2)}$ and $\alpha R$ on $e_{(2,0)}$ (Fig. 1). This configuration acts as a voltage divider circuit where the voltage drop across $R, V_{(1,2)}^{A}(1)=v_{0} \frac{R}{R+\alpha R}=v_{0} \frac{1}{1+\alpha}$. Tile A has output nodes $\{1,2\}$ across resistor $R$, whose voltage difference $V_{(1,2)}^{A}(1)$ is compared to the threshold $\tau$ to deter-

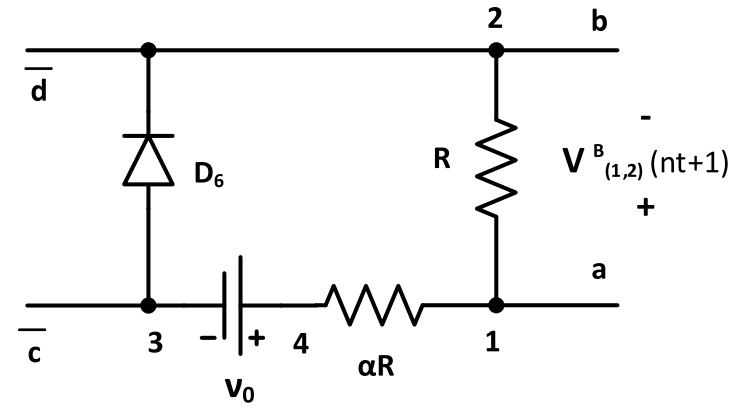

FIG. 2. Tile B (replicating seed tile) for the rcTAM. It has a voltage source of $\nu_{0}$, resistors $R$ and $\alpha R$, and an ideal diode $D_{6}$. It has one output node pair $\{1,2\}$ across $R$ and one input node pair $\{3,2\}$ across $D_{6}$. Node 1 has glue $a$, node 2 has glues $(b$ and $\bar{d})$, and node 3 has glue $\bar{c}$.

mine eligibility for further growth. Tile A has glues $g(1)=a$ and $g(2)=b$.

Tile B serves as the seed for replicated ladders, and is at position $k=n t+1$ after $n$ rounds of replication. Tile $\mathrm{B}$ has the same value for its voltage source $V_{(4,3)}^{B}(n t+1)=v_{0}$ as tile A, with resistor $R$ on $e_{(1,2)}, \alpha R$ on $e_{(1,4)}$, and ideal diode $D_{6}$ on $e_{(3,2)}$ with $V_{\mathrm{thr}}=0$. The diode is connected such that it is reverse biased and acts as an open circuit (Fig. 2). The polarity of the voltage across this open circuit is opposite to that required for attachment to tile $C$ at nodes $\{3,4\}$. It has one pair of output nodes $\{1,2\}$ across the resistor $R$, and one pair of input nodes $\{3,2\}$ across the diode $D_{6}$. Because $D_{6}$ is open circuited, $V_{(1,2)}^{B}$ is zero, and hence it is not eligible for attachment. Tile B has glues $g(2)=\{b, \bar{d}\}$ (two glues attached), $g(3)=\bar{c}$, and $g(1)=a$.

Tile $\mathrm{C}$ is the primary tile that contributes to growth of a ladder and determines whether replication occurs. Tile $\mathrm{C}$ consists of one dependent voltage source $V_{x}$ on $e_{(6,2)}$, three resistors with $R$ on $e_{(1,2)}, \alpha R$ on $e_{(2,7)}$, and $\beta R(\beta \gg 0)$ on $e_{(1,5)}$, and five diodes $\left(D_{3}\right.$ on $e_{(3,4)}, D_{1}$ on $e_{(6,5)}, D_{2}$ on $e_{(1,4)}, D_{4}$ on $e_{(5,3)}$, and $D_{5}$ on $e_{(4,3)}$, parallel to $\left.D_{3}\right)$, as shown in Fig. 3. Here, the dependent voltage source, $V_{x}=\frac{2 \tau v_{0}}{v_{0}}=2 \tau$ is activated only when connected to a circuit with $v_{0}$ (tile A or B). This tile has two pairs of output nodes $\{1,2\}$ and $\{3,4\}$, one pair of input nodes $\{1,7\}$, and has $g(1)=\{a, \bar{a}\}, g(2)=b, g(3)=c$, $g(4)=d$, and $g(7)=\bar{b}$. The voltage $V_{(1,2)}^{C}(k)$ depends on voltage division between $R$ and $\alpha R$. Resistor $\beta R$ has a large value so that $V_{x}$ has a minimal impact of $V_{x} \frac{1}{1+\beta}$ on $V_{(1,2)}^{C}(k)$ and thus on growth. Four diodes are connected across resistor $\beta R$. Diode $D_{2}$ and diode $D_{4}$ electrically isolate a replicated assembly from current in resistor $\beta R$, and vice versa. Diodes $D_{3}$ and $D_{5}$ are connected in parallel with opposite polarity between nodes 3 and node 4 . All diodes except $D_{3}$ have $V_{\text {thr }}=0$. Diode $D_{3}$ has $V_{\text {thr }}=2 \tau$, so that it is an open circuit throughout the growth process, with a voltage $V_{(3,4)}^{C}(k)$ equal to that across resistor $\beta R$. When replication occurs, diode $D_{5}$ completes the open loop connection across diode $D_{6}$ of tile $\mathrm{B}$ so that current can flow. $V_{(3,4)}^{C}$ thus determines whether replication will occur or not. 


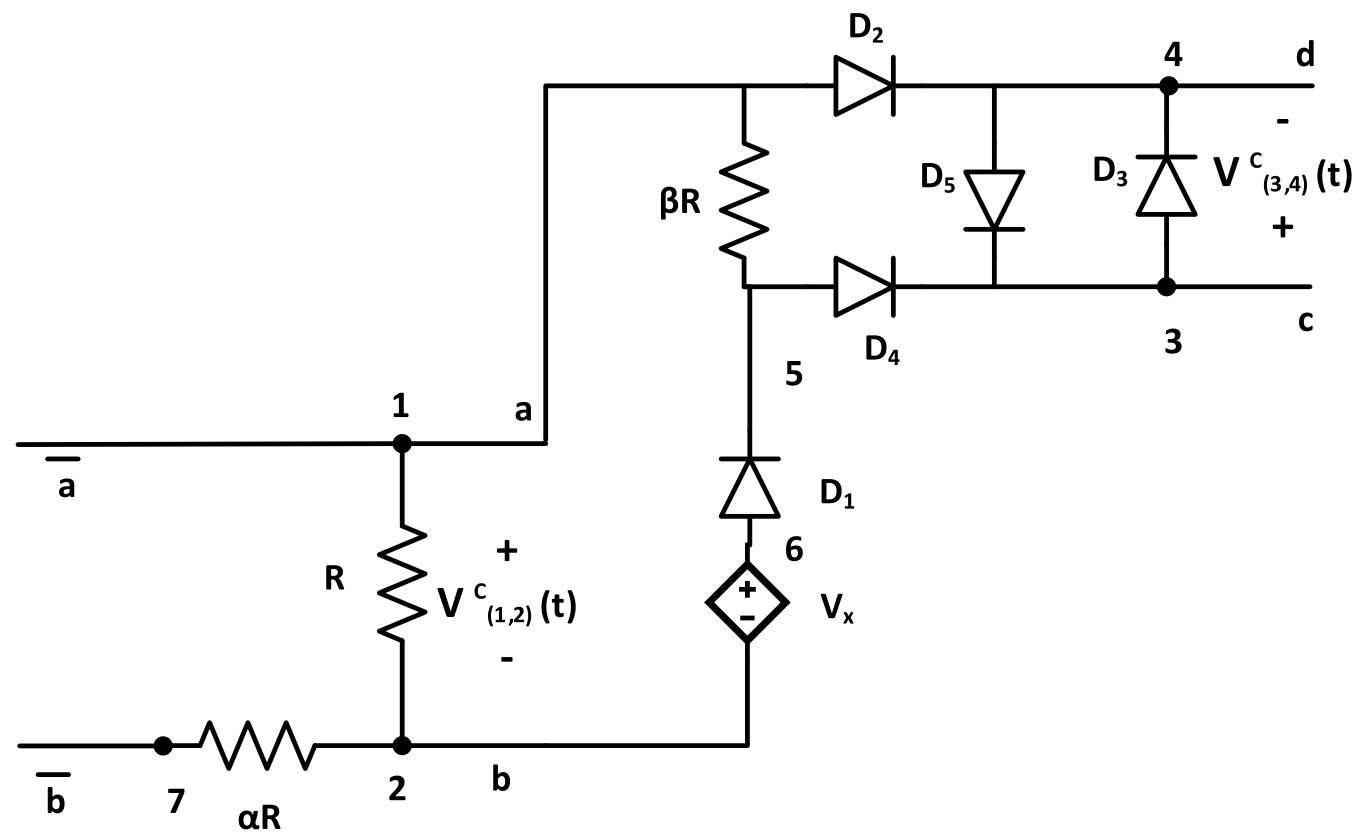

FIG. 3. Tile C (circuit tile) for the rcTAM consisting of three resistors $R, \alpha R$, and $\beta R$. Diode $D_{3}$ has $V_{\text {thr }}=2 \tau$, and $D_{1}, D_{2}, D_{4}, D_{5}$ have $V_{\mathrm{thr}}=0$. It has two pairs of output nodes at $\{1,2\}$ and $\{3,4\}$. The input node pair is $\{1,7\}$. Node 1 has two glues: $a$ and $\bar{a}$. Node 2 , node 3 , node 4 , and node 7 have glues $b, c, d$, and $\bar{b}$, respectively.

\section{SELF-REPLICATION}

The rcTAM has two types of tile attachments: one for regular growth and another for replication. The desired behavior of the assembly process is shown in Fig. 4. Assume the system has one seed tile A, multiple tiles B, and multiple tiles $\mathrm{C}$. As will be shown later, unwanted attachments between tiles that are not connected to a seeded ladder assembly do not occur. For regular growth, we must have $V_{(1,2)}^{A}(1) \geqslant \tau$ for tile A. Tile $\mathrm{C}$ attaches to tile A. As long as $V_{(1,2)}^{C}(k) \geqslant \tau, k \geqslant 2$, growth of the ladder proceeds with tile $C$ attaching to tip tile $C$ in the ladder. Eventually, as $v_{0}$ is dissipated by the voltage divider in the ladder, $V_{(1,2)}^{C}(k)<\tau$ (for example $k=t=3$ ) for the last tile $\mathrm{C}$ in the ladder (Fig. 4). At this point, as will be shown below, with a proper choice of $V_{x}, \alpha$, and $\beta, V_{x}$ will forward bias $D_{1}$ and drop enough voltage across $\beta R$ so that $V_{(3,4)}^{C}(t=$ $3) \geqslant \tau$. Tile B will then attach at nodes $\{3,4\}$ of tile $\mathrm{C}$, causing $D_{5}$ to become forward biased, providing a conducting path so that current flows through $R$ in tile $\mathrm{B}$ and thus growth of a new ladder starts. Diodes $D_{2}$ and $D_{4}$ are ideal diodes that are used to isolate the parent circuit from the child circuit. Thus, for replication to occur, the circuit needs to undergo a conformation change from an open circuit to a closed circuit. In summary, when $V_{(1,2)}^{C}\left(k_{\tau}\right) \geqslant \tau$, then $V_{(3,4)}^{C}\left(k_{\tau}\right)<\tau$ and only regular growth occurs. When $V_{(1,2)}^{C}(t)<\tau$, then $V_{(3,4)}^{C}(t) \geqslant \tau$, and replication growth occurs, only once per parent ladder assembly.

The ideal growth regions are depicted in Fig. 5. To summarize: when $V_{(1,2)}^{C}\left(k_{\tau}\right) \geqslant \tau$, then regular growth occurs where

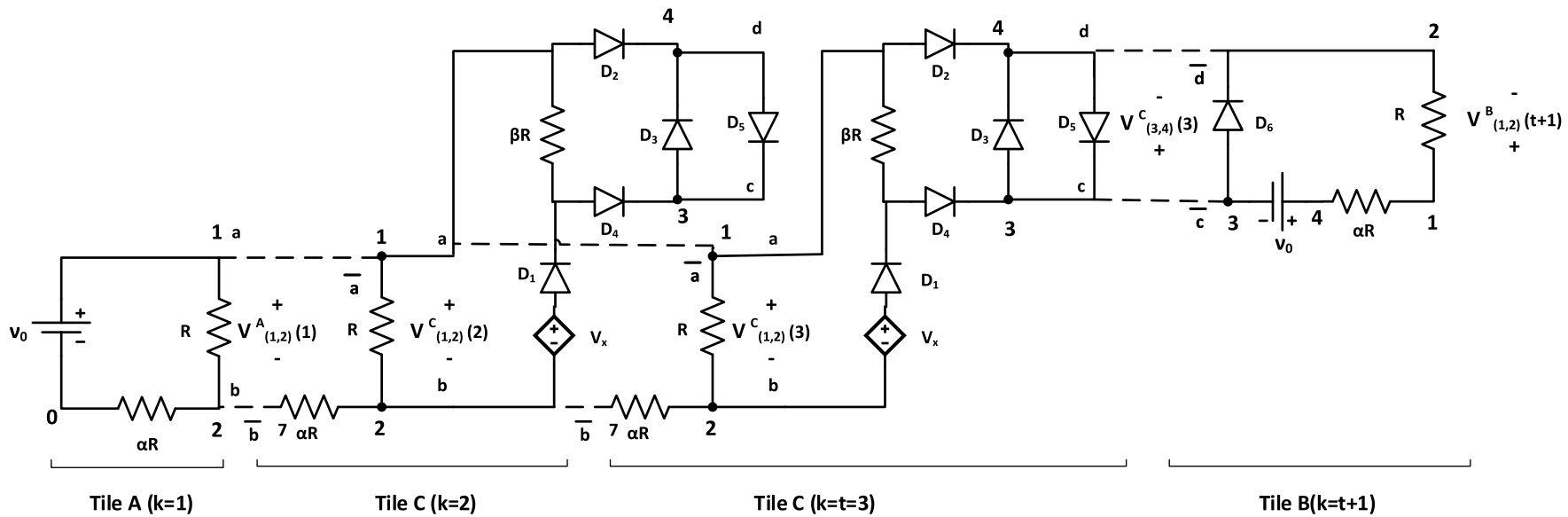

FIG. 4. Example of an assembly process. Here, an assembly starts from the seed tile A, and then two tile C's are attached to the seeded growth. As $V_{(1,2)}^{C}(3)$ falls below $\tau$, a tile B is attached to start replication. Attachments between tiles are shown as dashed lines. 




FIG. 5. The regions for regular growth and replication. When $V_{(1,2)}^{C}\left(k_{\tau}\right) \geqslant \tau$, regular growth occurs as $V_{(3,4)}^{C}\left(k_{\tau}\right)<\tau$. When $V_{(1,2)}^{C}\left(k_{\tau}\right)<\tau, V_{(3,4)}^{C}\left(k_{\tau}\right) \geqslant \tau$ and self-assembly starts replication.

$V_{(3,4)}^{C}\left(k_{\tau}\right)<\tau$; when $V_{(1,2)}^{C}\left(k_{\tau}\right)<\tau, V_{(3,4)}^{C}\left(k_{\tau}\right) \geqslant \tau$ and replication growth occurs; and when $V_{(1,2)}^{C}\left(k_{\tau}\right)>2 \tau$, the diode $D_{1}$ is reverse biased and, in turn, $V_{(3,4)}^{C}\left(k_{\tau}\right)=0$. Moreover, replication occurs only once at the maximum size of the ladder, $t$.

The goal of this section is to prove the following theorem in several stages.

Theorem III.1. There exists an infinite number of rcTAM systems exhibiting self-controlled regular growth, followed by replication in which this cycle repeats infinitely often.

As mentioned above, for the self-replication process to proceed as desired, certain attachments must be prevented, as described next.

Lemma III.1.1. Only attachments between rcTiles and a seeded ladder assembly (one containing tile A) are possible.

Proof. If tile $\mathrm{C}$ is not attached to a seeded assembly, because $V_{x}$ is a source dependent on being in a circuit connected to $v_{0}, V_{(1,2)}^{C}(k)=0<\tau$ and $V_{(3,4)}^{C}(k)=0<\tau$, and no attachments can occur between two unseeded tiles C's. Unless tile B is part of a circuit with tile $\mathrm{A}, D_{6}$ is reverse biased and an open circuit, and no current flows in $R$. Thus, $V_{(1,2)}^{B}(k)=0<\tau$, and no attachments occur between tiles $\mathrm{B}$ and $\mathrm{C}$. In addition, the polarity of $V_{(3,2)}$ in tile $\mathrm{B}$ is opposite to that required for attachment to nodes $\{3,4\}$ of tile $C$.

Lemma III.1.2. If $\nu_{0} /(1+\alpha) \geqslant \tau$, at least one tile $\mathrm{C}$ attaches to tile A.

Proof. For minimal growth, at least one tile $\mathrm{C}$ should attach to seed tile A. Therefore tile A must satisfy $V_{(1,2)}^{A}(1) \geqslant \tau$. According to Kirchoff's voltage law (KVL) [22] for the seed tile $\mathrm{A}(k=1$, Fig. 1$)$,

$$
\nu_{0}=V_{(1,2)}^{A}+V_{(2,0)}^{A} .
$$

Using Ohm's law $V_{(1,2)}^{A}=I R$ [22], where $I$ is the current, in Eq. (1) yields

$$
v_{0}=I R+\alpha I R, \quad v_{0}=V_{(1,2)}^{A}+\alpha V_{(1,2)}^{A} .
$$

Thus, $V_{(1,2)}^{A}=v_{0} /(1+\alpha)$ and tile $\mathrm{C}$ can attach to the seed.

To ensure regular growth and replication, a suitable value of $V_{x}$ needs to be determined.

Lemma III.1.3. If $V_{x}=2 \tau$ and $V_{(1,2)}^{C}(k)<\tau$, then $V_{(3,4)}^{C}(k)>\tau$.

Proof. If $V_{(1,2)}^{C}(k)<\tau$, then, regular growth has ceased. Since $V_{x}=2 \tau>V_{(1,2)}^{C}(k), D_{1}$ is forward biased $\left(V_{D_{1}}=0\right.$.) Applying KVL [22] to the loop $\left(V_{x}, D_{1}, \beta R, R\right)$ yields

$$
\begin{aligned}
V_{x}-V_{D_{1}}-V_{\beta R}-V_{R} & =0, \\
V_{x}-0-V_{(3,4)}^{C}-V_{(1,2)}^{C} & =0, \\
V_{(3,4)}^{C} & =V_{x}-V_{(1,2)}^{C} .
\end{aligned}
$$

Since $V_{x}=2 \tau$, then

$$
V_{(3,4)}^{C}(k)=2 \tau-V_{(1,2)}^{C}(k),
$$

Now $V_{(1,2)}^{C}(k)<\tau$ gives the conclusion $V_{(3,4)}^{C}(k)>\tau$.

Lemma III.1.4. If $V_{x}=2 \tau$ and $V_{(1,2)}^{C}(k)>2 \tau$, then $V_{(3,4)}^{C}(k)<\tau$.

Proof. Since $V_{(1,2)}^{C}(k)>V_{x}=2 \tau, D_{1}$ is reverse biased, no current flows through $\beta R$ and $V_{(3,4)}^{C}(k)=0<\tau$.

Lemma III.1.5. If $V_{x}=2 \tau$ and $\tau<V_{(1,2)}^{C}(k) \leqslant 2 \tau$, then $V_{(3,4)}^{C}(k)<\tau$.

Proof. Since $\tau<V_{(1,2)}^{C}(k) \leqslant V_{x}=2 \tau, D_{1}$ is forward biased and current flows in $\beta R$, hence $V_{(3,4)}^{C}(k) \neq 0$. Again, substituting in $\tau<V_{(1,2)}^{C}(k) \leqslant 2 \tau$ into equality (2) above gives

$$
0 \leqslant V_{(3,4)}^{C}(k)<\tau,
$$

where $V_{(3,4)}^{C}(k)=0$ when $V_{(1,2)}^{C}(k)=2 \tau$. In this case, $D_{1}$ is forward biased, but no current flows in $\beta R$.

Lemma III.1.6. If $V_{x}=2 \tau$ and $V_{(1,2)}^{C}(k)=\tau$, then $V_{(3,4)}^{C}(k)=\tau$.

Proof. Since $V_{(1,2)}^{C}(k)=\tau<V_{x}, D_{1}$ is forward biased, current flows through $\beta R$ and $V_{(3,4)}^{C}(k) \neq 0$. Substituting in $V_{(1,2)}^{C}(k)=\tau$ into Eq. (2) gives

$$
V_{(3,4)}^{C}(k)=\tau .
$$

Lemmas III.1.3-III.1.6 altogether imply that the desired behavior of the rcTAM is obtained, except when $V_{(1,2)}^{C}(k)=\tau$ (Lemma III.1.6). In that case, undesirably, both regular growth and replication will occur. Therefore, the model parameters should be chosen such that $V_{(1,2)}^{C}(k) \neq \tau$ for all $k$. Moreover, when $\tau \leqslant V_{(1,2)}^{C}(k) \leqslant 2 \tau$, diode $D_{1}$ is forward biased and it creates a closed loop among $V_{x}, D_{1}, \beta R$, and $R$ that would inject an additional current $I_{d}=2 \tau /(R+\beta R)$ into $R$. Depending on the value of resistor $\alpha R$, there could be several loops where diode $D_{1}$ is forward biased, and these additional $I_{d}$ currents could influence the final size of the ladder assembly. Finally, since tiles are added in parallel to previous tiles, the equivalent resistance between nodes $\{1,2\}$ could decrease according to Rayleigh's monotonicity law [23], which could cause $V_{(1,2)}$ to decrease below $\tau$ as more tiles attach. These potential problems, however, can be avoided if we chose $\alpha$ such that the voltage in the last two tiles added, $V_{(1,2)}^{C}(n t)$ and $V_{(1,2)}^{C}(n t-1)$, go from at least $2 \tau$ to under $\tau$ with the attachment of one tile. Therefore, for a ladder assembly of 
length $t$, the goal is to find some combination of $\nu_{0}, \tau$, and $\alpha$ such that $V_{(1,2)}^{C}(n t)<\tau$ for the last tile, and $V_{(1,2)}^{C}(n t-1) \geqslant$ $2 \tau$ for the next to last tile. In that case, current would only flow in $\beta R$, producing a $V_{(3,4)}^{C}(k) \neq 0$ for only the last tile at position $n t$. This also assures that only the last tile in an adult ladder at $n t$ will have $V_{(1,2)}^{C}(k)<\tau$.

An exact formula for the potential distribution of a onedimensional ladder has been derived in [20] as

$$
\begin{gathered}
V_{(1,2)}^{C}(n t)=\frac{\nu_{0}(-1)^{n t}}{A_{n t}+A_{n t-1}}=\frac{\nu_{0}}{\left|A_{n t}+A_{n t-1}\right|} \geqslant 0, \\
V_{(1,2)}^{C}(k)=v_{0}(-1)^{k} A_{k-1} \sum_{j=0}^{n t-k} \frac{1}{A_{k+j-1} A_{k+j}} \geqslant 0
\end{gathered}
$$

for every node $1 \leqslant k<n t$, where $A_{k}=\frac{\rho^{2 k+2}-1}{\rho^{k}\left(\rho^{2}-1\right)}$ and $\rho=$ $\frac{-(2+\alpha+\sqrt{\alpha(\alpha+4)})}{2}<-1$ for $\alpha>0$.

Lemma III.1.7. For a ladder assembly of size $t>0$, there exist $v_{0}, \tau$, and $\alpha>0$ such that $V_{(1,2)}^{C}(n t-1) \geqslant 2 \tau$ and $V_{(1,2)}^{C}(n t)<\tau$.

Proof. Using Eq. (3) for the last tile, the desired condition is equivalent to

$$
\begin{aligned}
& V_{(1,2)}^{C}(n t)<\tau, \\
& \frac{v_{0}(-1)^{n t}}{A_{n t}+A_{n t-1}}<\tau, \\
&\left|A_{n t}+A_{n t-1}\right|>\frac{v_{0}}{\tau} .
\end{aligned}
$$

The value of $\left|A_{n t}+A_{n t-1}\right|$ is

$$
\begin{aligned}
\left|A_{n t}+A_{n t-1}\right| & =\left|\frac{\rho^{2 n t+2}-1}{\rho^{n t}\left(\rho^{2}-1\right)}+\frac{\rho^{2 n t}-1}{\rho^{n t-1}\left(\rho^{2}-1\right)}\right| \\
& =\left|\frac{\rho^{2 n t+1}(1+\rho)-(1+\rho)}{\rho^{n t}\left(\rho^{2}-1\right)}\right| \\
& =\left|\frac{\left(\rho^{2 n t+1}-1\right)(1+\rho)}{\rho^{n t}(1+\rho)(\rho-1)}\right| \\
& =\left|\frac{\left(\rho^{2 n t+1}-1\right)}{\rho^{n t}(\rho-1)}\right|, \\
\left|A_{n t}+A_{n t-1}\right| & =\left|\frac{\rho^{n t+1}}{\rho-1}-\frac{1}{\rho^{n t}(\rho-1)}\right|>\frac{v_{0}}{\tau} .
\end{aligned}
$$

Therefore, it suffices to ensure that

$$
\left|\frac{\rho^{n t+1}}{\rho-1}\right|>\frac{v_{0}}{\tau}
$$

Next, further constraints for the condition $V_{(1,2)}^{C}(n t-1) \geqslant$ $2 \tau$ are determined. According to Rayleigh's monotonicity principle, voltages only decrease as the ladder grows, which guarantees that $V_{(1,2)}^{C}\left([n t-1]_{\tau}\right) \geqslant 2 \tau . V_{(1,2)}^{C}(n t-1)$ can be calculated using Eq. (4):

$$
\begin{aligned}
V_{(1,2)}^{C}(n t-1) & \geqslant 2 \tau, \\
v_{0}(-1)^{n t-1} A_{n t-2}\left[\frac{1}{A_{n t-2} A_{n t-1}}+\frac{1}{A_{n t-1} A_{n t}}\right] & \geqslant 2 \tau, \\
v_{0}(-1)^{n t-1}\left[\frac{A_{n t}+A_{n t-2}}{A_{n t} A_{n t-1}}\right] & \geqslant 2 \tau .
\end{aligned}
$$

Since

$$
\begin{aligned}
A_{n t}+A_{n t-2} & =\frac{\rho^{2 n t+2}-1}{\rho^{n t}\left(\rho^{2}-1\right)}+\frac{\rho^{2 n t-2}-1}{\rho^{n t-2}\left(\rho^{2}-1\right)} \\
& =\frac{\rho^{2 n t+2}-1+\rho^{2 n t}-\rho^{2}}{\rho^{n t}\left(\rho^{2}-1\right)} \\
& =\frac{\left(\rho^{2 n t}-1\right)\left(1+\rho^{2}\right)}{\rho^{n t}\left(\rho^{2}-1\right)}
\end{aligned}
$$

and

$$
A_{n t} \cdot A_{n t-1}=\left[\frac{\rho^{2 n t+2}-1}{\rho^{n t}\left(\rho^{2}-1\right)}\right] \times\left[\frac{\rho^{2 n t}-1}{\rho^{n t-1}\left(\rho^{2}-1\right)}\right],
$$

the term in square brackets in the inequality (6) can be reduced to

$$
\frac{A_{n t}+A_{n t-2}}{A_{n t} \cdot A_{n t-1}}=\frac{\left(\rho^{4}-1\right)\left(\rho^{n t-1}\right)}{\rho^{2 n t+2}-1},
$$

where, $\rho=\frac{-(2+\alpha+\sqrt{\alpha(\alpha+4)})}{2}$.

Further, since $\rho^{2 n t+2} \gg 1$, a sufficient condition for the second inequality can be further simplified (by ignoring the -1 in the denominator) to

$$
v_{0}(-1)^{n t-1}\left[\frac{\left(\rho^{4}-1\right)\left(\rho^{n t-1}\right)}{\rho^{2 n t+2}}\right] \geqslant 2 \tau .
$$

From [20], $\rho<-1$ and $\rho^{4}$ is a positive number. The value of $n t$ is odd if and only if both $n$ and $t$ are odd numbers. Otherwise, $n t$ is an even number. Therefore, there are two cases to consider for the length of the ladder $n t$ : even or odd. If $n t$ is odd, $(-1)^{n t-1}=+1, \rho^{n t-1}$ is a positive number, and $\rho^{2 n t+2}$ is also positive. So, $\frac{(-1)^{n t-1}\left(\rho^{4}-1\right)\left(\rho^{n t-1}\right)}{\rho^{2 n t+2}}$ is a positive number. On the other hand, if $n t$ is even, $(-1)^{n t-1}=-1$, $\rho^{n t-1}$ is a negative number, and their product in the numerator is positive. Moreover, $\rho^{2 n t+2}$ is a positive value. Therefore, $\frac{(-1)^{n t-1}\left(\rho^{4}-1\right)\left(\rho^{n t-1}\right)}{\rho^{2 n t+2}}$ is a positive number.

Therefore, inequality (7) is equivalent to the following inequalities:

$$
\begin{aligned}
& \nu_{0}\left(|\rho|^{4}-1\right) \frac{\left|\rho^{n t-1}\right|}{\left|\rho^{2 n t+2}\right|} \geqslant 2 \tau, \\
& \frac{\nu_{0}}{2 \tau}\left(|\rho|^{4}-1\right) \geqslant \frac{\left|\rho^{2 n t+2}\right|}{\left|\rho^{n t-1}\right|}, \\
& |\rho|^{n t+3} \leqslant \frac{\nu_{0}}{2 \tau}\left(|\rho|^{4}-1\right),
\end{aligned}
$$

and

$$
\frac{|\rho|^{n t+3}}{|\rho|^{4}-1} \leqslant \frac{\nu_{0}}{2 \tau} .
$$

Combining inequalities (5) and (8), a sufficient condition for self-replication is thus

$$
\frac{2|\rho|^{n t+3}}{|\rho|^{4}-1} \leqslant \frac{v_{0}}{\tau}<\left|\frac{\rho^{n t+1}}{\rho-1}\right| .
$$

Therefore, it suffices to ensure that

$$
\frac{2|\rho|^{n t+3}}{|\rho|^{4}-1}<\left|\frac{\rho^{n t+1}}{\rho-1}\right|
$$




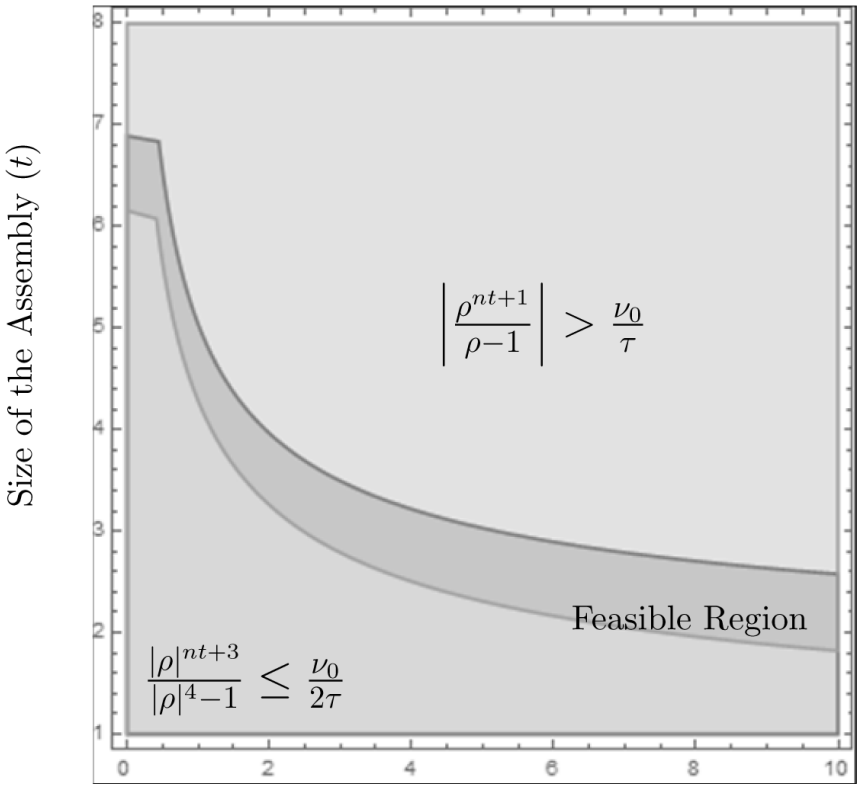

$\alpha$

FIG. 6. Feasible region for inequalities (5) and (8) for $v_{0}=100$ and $\tau=1$. The $y$ axis indicates the size of a terminal assembly and the $x$ axis indicates the value of $\alpha$.

so that there will exist a $\rho$ that satisfies both inequalities (5) and (8) for a given $\frac{\nu_{0}}{\tau}$. This condition is readily equivalent to

$$
0<\left|\rho^{4}\right|-2\left|\rho^{3}\right|+2\left|\rho^{2}\right|-1,
$$

which is true for all $|\rho|>1$, i.e., there is no restriction on $\rho$.

This argument completes the proof of Theorem III.1.

The range of parameters afforded by condition (10), is illustrated in Fig. 6 for $\nu_{0}=100$ and $\tau=1$. Circuit simulations of $V_{(1,2)}^{C}(k)$ using MATLAB-SIMULINK [24] show that, for given $\alpha, \tau$, and $\nu_{0}$ in the feasible region in Fig. 6, the voltages $V_{(1,2)}^{C}(n t-1) \geqslant 2 \tau$ and $V_{(1,2)}^{C}(n t)<\tau$. Therefore, appropriate input parameters can always be found so that $V_{(1,2)}^{C}(k) \neq \tau$, for example, $v_{0}=100 \mathrm{~V}, \tau=1 \mathrm{~V}$, and $\alpha=4$.

\section{A. Bounded growth for the ladders}

The cTAM model exhibits self-assembly with instant cooperation and control on the size of the assembly $[19,20]$. The maximum size depends on the voltage source $\left(v_{0}\right)$ of the seed tiles $A$ and $B$, the threshold voltage $(\tau)$, and the ratio of the resistor values in the tile set, $\alpha$. We can derive a similar bound for the rcTAM as shown next. In the rcTAM, because both seeds, tiles A and B, contain identical voltage sources and resistors, and each ladder is isolated from each other by reversed biased diodes, all copies of the ladder are equivalent. Therefore, the bound is proved for a single ladder.

Theorem III.2. The maximum length of the rcTAM ladder is bounded by

$$
B=\left\lceil\frac{\ln \left[\frac{\nu_{0}}{\tau} \frac{\beta}{\alpha+\beta+\alpha \beta}\right]}{\ln (1+\alpha)}+1\right\rceil .
$$

Proof. The voltage supply $v_{0}$ acts as a finite source of energy that is depleted as the circuit grows. The growth will stop when the $V_{(1,2)}^{C}(t)$ drops below a predefined threshold value. Using some basic electrical engineering methods, such as Kirchoff's voltage law [22], the maximum length $t$ of a self-assembled ladder is bounded from above. To calculate the equivalent resistance, each circuit tile has resistor $R$ in series with another resistor $\alpha R$, and that combined resistance is in parallel with $R$ of the previous circuit tile. If diode $D_{1}$ is forward biased, then the resistor $\beta R$ is in parallel with resistor $R$, whose equivalent resistance is denoted as $\gamma R$ where $\gamma=\frac{\beta}{1+\beta} \cdot V_{(1,2)}^{C}(n t)$ can be calculated in terms of $V_{(1,2)}^{C}(n t-1)$ as follows:

$$
V_{(1,2)}^{C}(n t)=V_{(1,2)}^{C}(n t-1)\left(\frac{\gamma R}{\gamma R+\alpha R}\right)+\frac{2 \tau R_{\mathrm{eq}}(n t)}{R_{\mathrm{eq}}(n t)+\beta R},
$$

where $R_{\mathrm{eq}}(n t)$ is the equivalent resistance seen by the dependent source $V_{x}$ from the rest of the ladder. Since $R>R_{\mathrm{eq}}(n t)$, Eq. (13) can be written as

$$
\begin{aligned}
& V_{(1,2)}^{C}(n t)<V_{(1,2)}^{C}(n t-1)\left(\frac{\gamma R}{\gamma R+\alpha R}\right)+\frac{2 \tau R}{(1+\beta) R}, \\
& V_{(1,2)}^{C}(n t)<V_{(1,2)}^{C}(n t-1)\left(\frac{\gamma}{\gamma+\alpha}\right)+\frac{2 \tau}{1+\beta} .
\end{aligned}
$$

By Lemma III.1.7, only the last tile will have a forward biased $D_{1}$ diode. All other tiles' diodes will be reverse biased. Hence, resistor $\beta R$ will not affect $V_{(1,2)}^{C}(k)$ for $(n-1) t+1 \leqslant k \leqslant$ $(n t-1)$. Thus,

$$
\begin{aligned}
V_{(1,2)}^{C}(n t-1)= & V_{(1,2)}^{C}(n t-2)\left(\frac{R_{\mathrm{eq}}(n t-1)}{R_{\mathrm{eq}}(n t-1)+\alpha R}\right), \\
V_{(1,2)}^{C}(n t)= & \left(V_{(1,2)}^{C}(n t-2)\right)\left(\frac{R_{\mathrm{eq}}(n t-1)}{R_{\mathrm{eq}}(n t-1)+\alpha R}\right) \\
& \times\left(\frac{\gamma}{\gamma+\alpha}\right)+\frac{2 \tau}{1+\beta},
\end{aligned}
$$

where $R_{\mathrm{eq}}(n t-1)$ indicates the equivalent resistance at tile position $(n t-1)$. Since $R_{\text {eq }}(n t-1)<R$ for any tile $k<n t$ because of the parallel combination of resistors, then substituting $R_{\mathrm{eq}}=R$ preserves the bound,

$$
V_{(1,2)}^{C}(n t)<\left(V_{(1,2)}^{C}(n t-2)\right)\left(\frac{1}{1+\alpha}\right)\left(\frac{\gamma}{\gamma+\alpha}\right)+\frac{2 \tau}{1+\beta} .
$$

Iterating the process recursively up to $k=(n-1) t+1$ yields

$$
V_{(1,2)}^{C}(n t)<v_{0}\left(\frac{1}{1+\alpha}\right)^{t-1}\left(\frac{\gamma}{\gamma+\alpha}\right)+\frac{2 \tau}{1+\beta} .
$$

Thus the growth will stop at a maximum size when $V_{(1,2)}^{C}(n t)<\tau$, i.e., when the following equivalent inequalities hold:

$$
\begin{aligned}
& v_{0}\left(\frac{1}{1+\alpha}\right)^{t-1}\left(\frac{\gamma}{\gamma+\alpha}\right)+\frac{2 \tau}{1+\beta}<\tau, \\
& v_{0}\left(\frac{1}{1+\alpha}\right)^{t-1}\left(\frac{\gamma}{\gamma+\alpha}\right)<\tau-\frac{2 \tau}{1+\beta},
\end{aligned}
$$




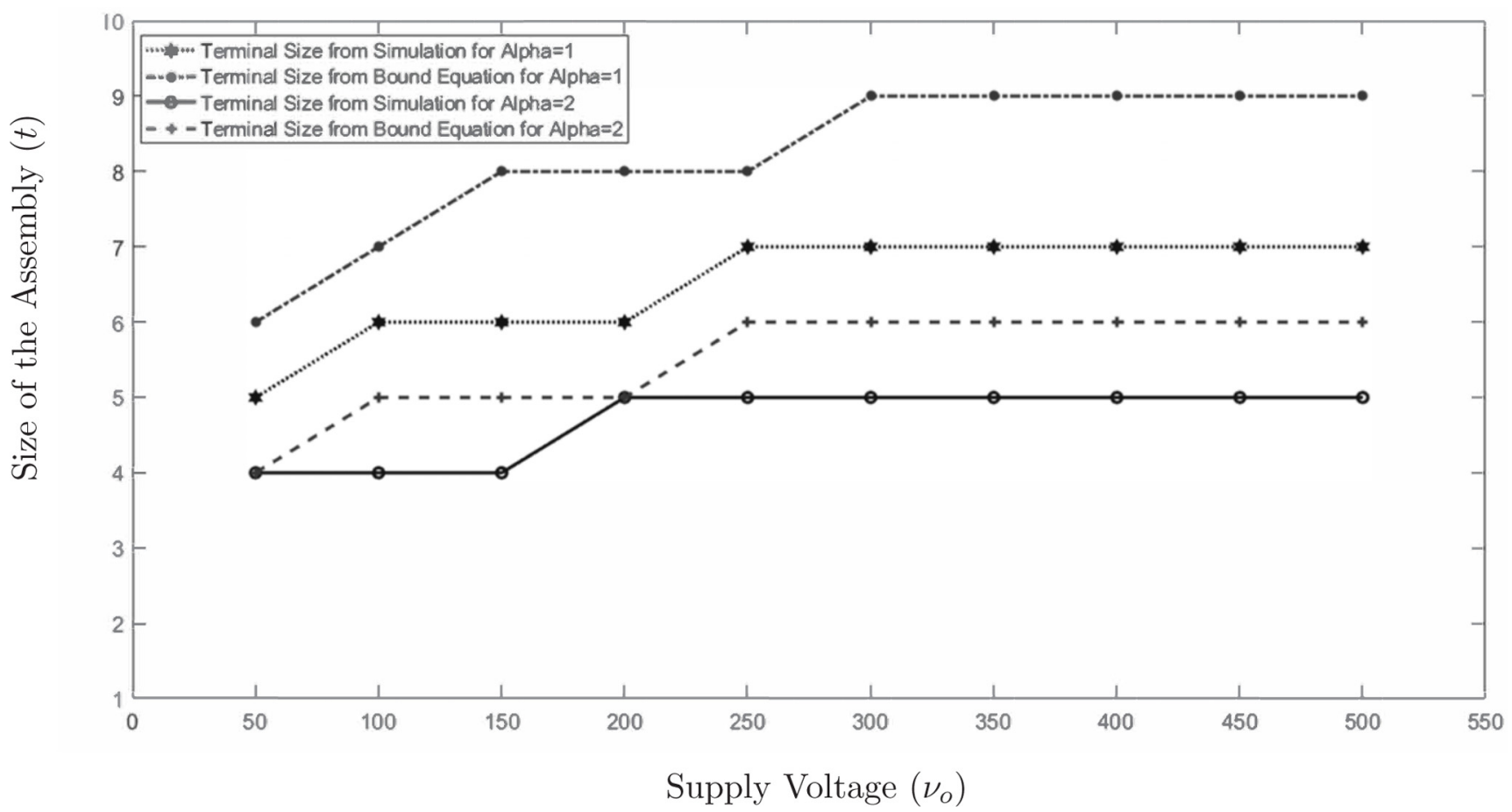

FIG. 7. Quality of the bound on ladder size for model parameters $\tau=1, n=1, \beta=1000$. This plot confirms the validity of the bound and how tight it is for values of $\alpha=1,2$. The simulations of the circuits were done with MATLAB-SIMULINK.

$$
\begin{aligned}
& \left(\frac{1}{1+\alpha}\right)^{t-1}<\frac{\tau-\frac{2 \tau}{1+\beta}}{v_{0}\left(\frac{\gamma}{\alpha+\gamma}\right)}, \\
& (1+\alpha)^{t-1}>\frac{\nu_{0}\left(\frac{\gamma}{\alpha+\gamma}\right)}{\tau\left(1-\frac{2}{1+\beta}\right)}, \\
& (t-1) \ln (1+\alpha)>\ln \left[\frac{\nu_{0}\left(\frac{\gamma}{\alpha+\gamma}\right)}{\tau\left(1-\frac{2}{1+\beta}\right)}\right], \\
& t>\frac{\ln \left[\frac{\nu_{0}\left(\frac{\gamma}{\alpha+\gamma}\right)}{\tau\left(1-\frac{2}{1+\beta}\right)}\right]}{\ln (1+\alpha)}+1, \\
& t>\frac{\ln \left[\frac{\nu_{0}\left(\frac{\gamma}{\alpha+\gamma}\right)}{\tau\left(\frac{\beta-1}{\beta+1}\right)}\right]}{\ln (1+\alpha)}+1, \\
& t>\frac{\ln \left[\left(\frac{\nu_{0}}{\tau}\right)\left(\frac{\gamma}{\alpha+\gamma}\right)\left(\frac{\beta+1}{\beta-1}\right)\right]}{\ln (1+\alpha)}+1 .
\end{aligned}
$$

Taking $\left(\frac{\beta+1}{\beta-1}\right) \approx 1$ for large $\beta$, the bound is preserved, and, substituting $\frac{\gamma}{\alpha+\gamma}=\frac{\beta}{\alpha+\beta+\alpha \beta}$, then

$$
t>\frac{\ln \left[\left(\frac{\nu_{0}}{\tau}\right)\left(\frac{\beta}{\alpha+\beta+\alpha \beta}\right)\right]}{\ln (1+\alpha)}+1 .
$$

From Theorem III.2, the effect of the additional circuit components to achieve replication is small $(<1)$, namely, an additive term $\left[\ln \left(\frac{\beta}{(\alpha+\beta+\alpha \beta}\right)\right]$. Figure 7 confirms the soundness of this bound. Here, the maximum size of a circuit tile assembly model is calculated both as the bound B in Eq. (12) and by using a simulation with MATLAB-SIMULINK [24]. The same procedure was done for values of $\alpha$ equal to 1 and 2 .

Therefore, an rcTAM generates a circuit assembly system that is self-controlled and self-replicated with identical copies. For example, consider a terminal circuit configuration for input parameters $v_{o}=50, \tau=1, \alpha=1$, and $\beta=1000$. This circuit ladder grows up to length $t=5$. Then $V_{(1,2)}^{C}(5)$ falls below $\tau$ and $V_{(3,4)}^{C}(5)$ becomes greater than $\tau$. A tile $\mathrm{B}$ is attached to the $V_{(3,4)}^{C}(5)$ node terminals and starts to replicate a copy of itself. Figure 8 shows $V_{(1,2)}^{k}$ for $1 \leqslant k \leqslant t$ and $t=5$. Four replications are considered, i.e., $n=5$. The pattern of voltages is the same in the original and replicated ladders.

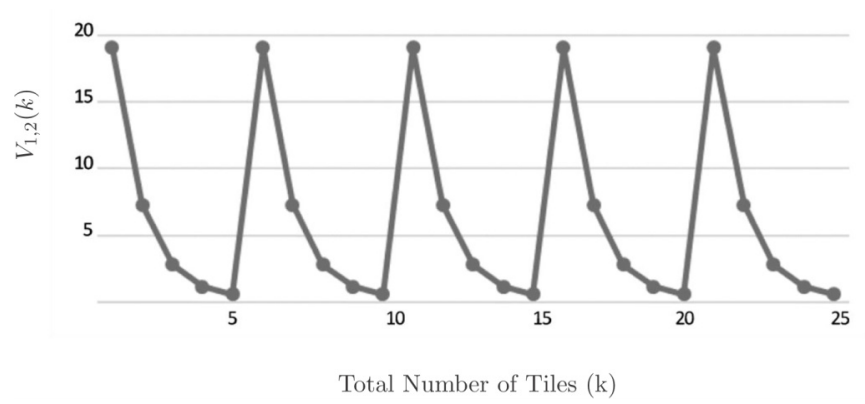

FIG. 8. $V_{(1,2)}(k)$ after tile attachment for model parameters $v_{0}=$ 50, $\tau=1, n=5, \alpha=1$, and $\beta=1000$. In this example, replication occurs after $t=5$ tile attachments. This figure illustrates that the pattern of $V_{(1,2)}(k)$ repeats identically after every replication. The $y$ axis shows the potential drop across the $R$ resistor for the terminal assembly. The $x$ axis indicates the total tile size of the assembly. After 25 steps, four replications have occurred. 


\section{B. Aging and the ecology of reTAMs}

Biological individuals do not replicate forever, but age and die. Likewise, electrical components, or wires for that matter, will not function indefinitely. As components age, they will experience performance degradation and eventual failure. For example, electromigration, a process that causes circuit failure by increasing the resistivity of a wire [25], is accelerated by high temperatures [26], [27]. The time to failure [25] is a common measure of reliability. In rcTAM, the maximum current will flow in resistor $\alpha R$ of either seed tile A or B. Therefore, these resistors are most likely to fail after some time $M$, the time to failure or lifetime of that component. Thus, this age-based failure mechanism ensures that the rcTAM will produce a stable population of ladders.

Theorem III.3. An rcTAM will produce a stable population of size $\left\lfloor\frac{M}{t}\right\rfloor$, where $\mathrm{M}$ indicates the lifetime of a resistor and $t$ is the number of time steps required for a ladder to reach adulthood (maximum size) and start to replicate.

Proof. Let $M$ be the time to failure of resistor $\alpha R$ in tile A or B. An rcTAM starts its growth from the tile A, and after a certain amount of time or number of attachments the growth stops at an adult assembly. After that, the replication process starts and the growth of the child ladder continues according to the replication dynamics of rcTAM. The number of time steps (tile attachments) for the ladders to become an adult again is $t$, the size of the adult ladders. Assuming that a tile attaches at the rate of 1 tile per time unit, for the original ladder to replicate we must have $M>t$. The resistor $\alpha R$ of tile $\mathrm{A}$ or $\mathrm{B}$ will fail after $M$ time steps, so the total living population has roughly $\frac{M}{t}$ ladders. When $\alpha R$ fails, that ladder becomes electrically inactive. On the other hand, if a replicated ladder has started growing, it will complete its life cycle and become an adult, replicating in turn. Therefore, the total population of ladders or the size of the assembly remains ultimately constant, with size $\left\lfloor\frac{M}{t}\right\rfloor$ complete ladders.

For example, if $M=15.5$ and $t=4$, the assembly has three mature ladders and one partial ladder when the first ladder dies. Nevertheless, the last ladder will complete the growth process and initiate another replication. This cycle repeats all over again. Thus the total living population stabilizes at size $\left\lfloor\frac{15.5}{4}\right\rfloor=3$.

\section{DISCUSSION AND CONCLUSION}

We have proposed the rcTAM model of self-assembly consisting of basic circuit components (resistors, diodes, and voltage sources) with a self-controlled growth mechanism. Except for the dependent source, the components do not include transistors and are not organic. The model exhibits instant cooperation among components that results in provable properties, such as self-controlled growth and self-replication. In addition, realistic failure mechanisms were used to prove that the system produces a stable population of adult assemblies. Therefore, the rcTAM is a family of models of nonbiological systems that exhibit lifelike properties, such as self-assembly, self-controlled growth, and controlled selfreplication. Usually, organic chemistry is assumed for lifelike systems (though not exclusively). Several researchers have been exploring inorganic chemical systems that have certain properties of biological life, including self-replication, and asking the question whether organic chemistry is a prerequisite to lifelike systems [11]. In that spirit, rcTAMs have something to offer. Given their simplicity, it is somewhat surprising that rcTAMs exhibit such seemingly advanced capabilities. This emphasizes, perhaps, the power of self-assembly. For example, would a static circuit in the rcTAM have the same properties if it did not self-assemble?

An open question is to characterize the computational power of the rcTAM model. Given its parentage in algorithmic self-assembly models where glues provide the computational power, with sufficient number and types of glues, rcTAM would also be Turing universal. The question is really whether the electrical mechanisms present in the model allow computational universality to be achieved with a small glue set. In recent work [28], one-dimensional resistive ladders with annealing schedules of a decreasing sequence of thresholds were found to be capable of super-Turing computation.

Although an abstract, theoretical model intended to explore mechanisms of self-replication, the rcTAM might have application in self-assembly of nanostructures in general. Electric phenomena are useful in a variety of artificial growth processes, such as additive manufacturing [29], electrospray technology [30], and other nanomanufacturing technologies [31-33]. Electrostatic interactions have been reported as a mechanism to control nanoscale assemblies [34-36], and the rcTAM affords a theoretical framework to explore the ability of potential fields to control self-assembly size and shape.

The model also might have relevance to several examples of biological function, self-assembly, and organization. For example, galvanotaxis is the movement of cells under the influence of an electric field, and is a mechanism for both wound healing as well as embryonic cell migration [37]. The biological molecules that inspire artificial approaches to self-assembly, namely lipids, nucleic acids, and amino acids, are charged molecules, and electric phenomena are directly involved in their formation and stability [38]. Electric circuits, like the Hodgkin-Huxley model, have been used to describe mechanisms that produce action potentials in cells, particularly neural [39].

As described in Sec. I, endogenous fields from potential distributions in collection of cells interact with genetic mechanisms for pattern formation during tissue regeneration or embryo development [12-16]. There are similarities between the cTAM models and the effects of these bioelectric networks. The cTAM is potentially an abstract model for how electric signals might propagate in bioelectric networks, and the rcTAM for how they might replicate. Both the cTAM models and models of bioelectric networks capture how electric potentials can influence shape, size, and pattern. Except for small frequency-dependent capacitive effects, the electrical connection between cells across the gap junction is primarily Ohmic [40], and thus, the resistive ladder of the rcTAM is an approximate equivalent circuit for bioelectric intercellular communication among cells in a network [41]. rcTAM and related models describe growing networks of circuit tiles whose properties are dynamic as the network structure changes. During processes like embryogenesis, networks of somatic cells are also dynamic, and thus the methods used to analyze 
cTAM networks might enable theoretical predictions to be made about them. In addition, in preliminary work [28], the cTAM is shown to be a powerful model of computation, and might elucidate the role of computation in bioelectric networks and information processing in biological systems. Therefore, the rcTAM and related models capture some of the characteristics of bioelectric networks, though abstracted to make analysis simpler, and that connection would be an interesting area for future investigation.

In conclusion, a tile assembly model, rcTAM, whose components are simple electric circuits, exhibits the lifelike properties of self-assembled and self-controlled growth with self-replication. It accomplishes this without explicit biological components or mechanisms. Nevertheless, there seem to be mechanisms that are necessary that have analogs in biology, namely self-assembly, a source of energy that is consumed to drive growth, a threshold value for control, and even, when replication occurs, conformational change. Thus, the rcTAM has potential not only to study self-assembly of nanostructures that are driven by an electric field, but also to provide insight into the necessary properties for systems to achieve characteristics similar to living systems.
[1] J. G. Miller, Living Systems (McGraw-Hill, New York, 1978).

[2] S. A. Kauffman, The Origins of Order: Self-Organization and Selection in Evolution (Oxford University, New York, 1993).

[3] J. Von Neumann and A. W. Burks, Theory of Self-Reproducing Automata (University of Illinois Press, 1966).

[4] G. M. Whitesides and M. Boncheva, Beyond molecules: Selfassembly of mesoscopic and macroscopic components, Proc. Natl. Acad. Sci. USA 99, 4769 (2002).

[5] M. A. Boles, M. Engel, and D. V. Talapin, Self-assembly of colloidal nanocrystals: From intricate structures to functional materials, Chem. Rev. 116, 11220 (2016).

[6] C. Mirkin, R. L. Letsinger, R. C. Mucic, and J. J. Storhoff, A DNA-based method for rationally assembling nanoparticles into macroscopic materials, Nature (London) 382, 607 (1996).

[7] E. Winfree, F. Liu, L. A. Wenzler, and N. C. Seeman, Design and self-assembly of two-dimensional DNA crystals, Nature (London) 394, 539 (1998).

[8] C. E. Shannon, Von neumann's contributions to automata theory, Bull. Am. Math. Soc. 64, 123 (1958).

[9] K. Drexler, Engines of Creation (Doubleday, New York, 1986).

[10] M. Neveu, H. J. Kim, and S. A. Brenner, The "strong" RNA world hypothesis: Fifty years old, Astrobiology 13, 391 (2013).

[11] L. Cronin and S. I. Walker, Beyond prebiotic chemistry, Science 352, 1174 (2016).

[12] M. Levin, Endogenous bioelectrical networks store non-genetic patterning information during development and regeneration, J. Physiol. 592, 2295 (2014).

[13] A. Pietak and M. Levin, Bioelectric gene and reaction networks: computational modelling of genetic, biochemical, and bioelectrical dynamics in pattern regulation, J. R. Soc. Interface 14, 20170425 (2017).

[14] M. Levin and C. J. Martyniuk, The bioelectric code: An ancient computational medium for dynamic control of growth and form, Biosystems 164, 76 (2018).

[15] J. Cervera, V. P. Pai, M. Levin, and S. Mafe, From non-excitable single-cell to multicellular bioelectrical states supported by ion channels and gap junction proteins: Electrical potentials as distributed controllers, Prog. Biophys. Mol. Biol. 149, 39 (2019).

[16] J. Cervera, J. A. Manzanares, S. Mafe, and M. Levin, Synchronization of bioelectric oscillations in networks of nonexcitable cells: From single-cell to multicellular states, J. Phys. Chem. B 123, 3924 (2019).
[17] R. Deaton, M. Garzon, R. Yasmin, and T. Moore, A model for self-assembling circuits with voltage-controlled growth, Int. J. Circuit Theory Appl. (2020), doi:10.1002/cta.2806.

[18] A. Pietak and M. Levin, Bioelectrical control of positional information in development and regeneration: A review of conceptual and computational advances, Prog. Biophys. Mol. Biol. 137, 52 (2018), special issue, Biological Challenges in Morphogenesis.

[19] R. Deaton, R. Yasmin, T. Moore, and M. Garzon, Selfassembled dc resistive circuits with self-controlled voltagebased growth, in International Conference on Unconventional Computation and Natural Computation, 16th International Conference, UCNC 2017, Fayetteville, AR, June 5-9, 2017, edited by M. J. Patitz and M. Stannett, Lecture Notes in Computer Science Vol. 10240 (Springer, Berlin 2017), pp. 129-143.

[20] Y. Yan, M. Garzon, and R. Deaton, Harmonic circuit selfassembly in CTAM models, IEEE Trans. Nanotechnol. 18, 195 (2018).

[21] Y. Yan, M. Garzon, and R. Deaton, Self-assembly of 2-D resistive electric grids, IEEE Trans. Nanotechnol. 18, 562 (2019).

[22] J. Nilsson, Engineering Circuit Analysis-William H. Hayt and Jack E. Kemmerly, IEEE Trans. Educ. 20, 121 (1977).

[23] P. G. Doyle and J. L. Snell, Random Walks and Electric Networks (Mathematical Association of America, Washington, DC, 1984).

[24] MATLAB, version 9.4.0.813654 (r2010a) (2018).

[25] V. Mishra and S. S. Sapatnekar, Probabilistic wire resistance degradation due to electromigration in power grids, IEEE Trans. Comput. Aided Des. Integr. Circuits Syst. 36, 628 (2017).

[26] F. Sinnadurai, P. Spencer, and K. Wilson, Some observations on the accelerated ageing of thick-film resistors, Act. Passive Electron. Compon. 6, 241 (1980).

[27] N. Sinnadurai and K. Wilson, The aging behavior of commercial thick-film resistors, IEEE Trans. Compon. Hybrids Manuf. Technol. 5, 308 (1982).

[28] M. Garzon, T. Moore, R. Deaton, and R. Yasmin, Computational power and complexity of self-assembly resistive electric ladders (unpublished).

[29] B. Y. Decker and Y. X. Gan, Electric field-assisted additive manufacturing polyaniline based composites for thermoelectric energy conversion, J. Manuf. Sci. Eng. 137, 024504 (2015).

[30] P. Z. El-Khoury, E. Khon, Y. Gong, A. G. Joly, P. Abellan, J. E. Evans, N. D. Browning, D. Hu, M. Zamkov, and W. P. Hess, Electric field enhancement in a self-assembled 
2D array of silver nanospheres, J. Chem. Phys. 141, 214308 (2014).

[31] O. Englander, D. Christensen, J. Kim, L. Lin, and S. J. Morris, Electric-field assisted growth and self-assembly of intrinsic silicon nanowires, Nano Lett. 5, 705 (2005).

[32] J. Gong and N. Wu, Electric-field assisted assembly of colloidal particles into ordered nonclose-packed arrays, Langmuir 33, 5769 (2017)

[33] D. A. Brown, J.-H. Kim, H.-B. Lee, G. Fotouhi, K.-H. Lee, W. K. Liu, and J.-H. Chung, Electric field guided assembly of one-dimensional nanostructures for high performance sensors, Sensors 12, 5725 (2012).

[34] M. Neveu, H. J. Kim, and S. A. Brenner, Electrostatic selfassembly of macroscopic crystals using contact electrification, Nat. Mater. 2, 241 (2003).

[35] D. A. Walker, B. Kowalczyk, M. O. de La Cruz, and B. A. Grzybowski, Electrostatics at the nanoscale, Nanoscale 3, 1316 (2011).

[36] E. B. Lindgren, I. N. Dervenev, A. Khachatourian, H.-K. Chen, A. J. Stace, and E. Besley, Electrostatic self-assembly: Un- derstanding the significance of the solvent, J. Chem. Theory Comput. 14, 905 (2018).

[37] R. Nuccitelli and C. A. Erickson, Embryonic cell motility can be guided by physiological electric fields, Exp. Cell. Research 147, 195 (1983).

[38] R. N. Pereira, B. W. Souza, M. A. Cerqueira, J. A. Teixeira, and A. A. Vicente, Effects of electric fields on protein unfolding and aggregation: Influence on edible films formation, Biomacromolecules 11, 2912 (2010).

[39] A. L. Hodgkin and A. F. Huxley, A quantitative description of membrane current and its application to conduction and excitation in nerve, J. Physiol. 117, 500 (1952).

[40] J. Cervera, S. Meseguer, and S. Mafe, The interplay between genetic and bioelectrical signalling permits a spatial regionalisation of membrane potentials in model multicellular assemblies, Sci. Rep. 6, 35201 (2016).

[41] E. C. Fear and M. A. Stuchly, A novel equivalent circuit model for gap-connected cells, Phys. Med. Biol. 43, 1439 (1998). 